\title{
Sets of exact approximation order by rational numbers III
}

\author{
Yann Bugeaud (Strasbourg) \& Carlos Gustavo Moreira (Rio de Janeiro)
}

\begin{abstract}
For a function $\Psi: \mathbf{R}_{>0} \rightarrow \mathbf{R}_{>0}$, let $\operatorname{Exact}(\Psi)$ be the set of real numbers that are approximable by rational numbers to order $\Psi$, but to no order $c \Psi$ with $0<c<1$. When $\Psi$ is non-increasing and satisfies $\Psi(x)=o\left(x^{-2}\right)$, we establish that Exact $(\Psi)$ has Hausdorff dimension $2 / \lambda$, where $\lambda$ is the lower order at infinity of the function $1 / \Psi$. Furthermore, we study the set $\operatorname{Exact}(\Psi)$ when $\Psi$ is not assumed to be non-increasing and show that the set $\operatorname{Exact}(\Psi)$ is uncountable for a large class of functions $\Psi$ satisfying $\Psi(x)=o\left(x^{-2}\right)$.
\end{abstract}

\section{Introduction}

For a function $\Psi: \mathbf{R}_{>0} \rightarrow \mathbf{R}_{>0}$, let

$$
\mathcal{K}(\Psi):=\left\{\xi \in \mathbf{R}:\left|\xi-\frac{p}{q}\right|<\Psi(q) \text { for infinitely many rational numbers } \frac{p}{q}\right\}
$$

denote the set of $\Psi$-approximable real numbers and let

$$
\operatorname{Exact}(\Psi):=\mathcal{K}(\Psi) \backslash \bigcup_{m \geq 2} \mathcal{K}((1-1 / m) \Psi)
$$

be the set of real numbers approximable to order $\Psi$ and to no better order. In other words, $\operatorname{Exact}(\Psi)$ is the set of real numbers $\xi$ such that

$$
|\xi-p / q|<\Psi(q) \quad \text { infinitely often }
$$

and

$$
|\xi-p / q| \geq c \Psi(q) \quad \text { for any } c<1 \text { and any } q \geq q_{0}(c, \xi),
$$

where $q_{0}(c, \xi)$ denotes a positive real number depending only on $c$ and on $\xi$. If $\Psi$ is nonincreasing and satisfies $\Psi(x)=o\left(x^{-2}\right)$, Jarník [11], Satz 6, used the theory of continued fractions to construct explicitly real numbers in $\mathcal{K}(\Psi)$ which do not belong to any set $\mathcal{K}(c \Psi)$ with $0<c<1$. His result can be restated as follows.

2010 Mathematics Subject Classification : 11J04. Keywords : Approximation by rational numbers, Hausdorff dimension. 
Theorem J. Let $\Psi: \mathbf{R}_{>0} \rightarrow \mathbf{R}_{>0}$ be a non-increasing function satisfying $\Psi(x)=o\left(x^{-2}\right)$. Then, the set $\operatorname{Exact}(\Psi)$ is uncountable.

In 1924, Khintchine [12] (see also his book [13]) used the theory of continued fractions to prove that, if $x \mapsto x^{2} \Psi(x)$ is non-increasing, then $\mathcal{K}(\Psi)$ has Lebesgue measure zero if the sum $\sum_{x \geq 1} x \Psi(x)$ converges and has full Lebesgue measure otherwise. In the convergence case, his result was considerably refined by Jarník who established [11], Satz 5, that, if $\Phi: \mathbf{R}_{>0} \rightarrow \mathbf{R}_{>0}$ is a positive continuous function such that $\Phi(x) / x$ tends monotonically to infinity with $x$, then the sets $\mathcal{K}(\Psi) \backslash \mathcal{K}(\Psi \circ \Phi)$ and $\mathcal{K}(\Psi)$ have the same Hausdorff $\mathcal{H}^{f}$ measure for a general dimension function $f$. We refer the reader to $[17,8]$ for background on the theory of Hausdorff measure. As usual, we denote by dim the Hausdorff dimension. Jarník's statement implies that

$$
\operatorname{dim} \mathcal{K}(\Psi)=\frac{2}{\lambda}
$$

where $\lambda$ denotes the lower order at infinity of the function $1 / \Psi$ and the lower order at infinity $\lambda(g)$ of a function $g: \mathbf{R}_{>0} \rightarrow \mathbf{R}_{>0}$ is defined by

$$
\lambda(g)=\liminf _{x \rightarrow+\infty} \frac{\log g(x)}{\log x} .
$$

This notion arises naturally in estimating the Hausdorff dimension of the sets $\mathcal{K}(\Psi)$, see e.g., Dodson [6] and Dickinson [5].

Jarník's result is, however, not strong enough to imply that $\operatorname{Exact}(\Psi)$ and $\mathcal{K}(\Psi)$ have the same Hausdorff dimension, a problem raised by Beresnevich, Dickinson and Velani at the end of [1].

Problem 1. Let $\Psi: \mathbf{R}_{>0} \rightarrow \mathbf{R}_{>0}$ be a non-increasing function satisfying $\Psi(x)=o\left(x^{-2}\right)$. To compute the Hausdorff dimension of $\operatorname{Exact}(\Psi)$.

Problem 1 was solved in [2] for a large class of functions $\Psi$.

Theorem B1. Let $\Psi: \mathbf{R}_{>0} \rightarrow \mathbf{R}_{>0}$ be such that $x \mapsto x^{2} \Psi(x)$ is non-increasing. Assume that the sum $\sum_{x \geq 1} x \Psi(x)$ converges. If $\lambda$ denotes the lower order at infinity of the function $1 / \Psi$, then

$$
\operatorname{dim} \operatorname{Exact}(\Psi)=\operatorname{dim} \mathcal{K}(\Psi)=\frac{2}{\lambda} .
$$

Up to the extra assumption on $\Psi$, namely the fact that $x \mapsto x^{2} \Psi(x)$ is non-increasing (which implies that $\Psi$ is decreasing), Theorem B1 provides a very satisfactory strengthening of Theorem J when the sum $\sum_{x \geq 1} x \Psi(x)$ converges. When this sum diverges, Problem 1 was investigated in 1952 by Kurzweil [14], a student of Jarník. Among other results, he established that the set $\mathcal{K}(3 \Psi) \backslash \mathcal{K}(\Psi)$ has full Hausdorff dimension for a large class of functions $\Psi$, but his method does not seem to yield any result on $\operatorname{Exact}(\Psi)$. The following statement, established in [3] following the method introduced in [2], answers Problem 1 for a class of functions $\Psi$ such that the sum $\sum_{x \geq 1} x \Psi(x)$ diverges. 
Theorem B2. Let $\Psi: \mathbf{R}_{>0} \rightarrow \mathbf{R}_{>0}$ be such that $x \mapsto x^{2} \Psi(x)$ is non-increasing. Assume that the sum $\sum_{x \geq 1} x \Psi(x)$ diverges and that, for any positive real number $\varepsilon$, we have

$$
\frac{1}{x^{2+\varepsilon}} \leq \Psi(x) \leq \frac{1}{100 x^{2} \log x},
$$

for any sufficiently large $x$. Then we have

$$
\operatorname{dim} \operatorname{Exact}(\Psi)=\operatorname{dim} \mathcal{K}(\Psi)=1 .
$$

One of the purposes of the present note is to extend Theorem B2 to all non-increasing functions $\Psi: \mathbf{R}_{>0} \rightarrow \mathbf{R}_{>0}$ such that $\Psi(x)=o\left(x^{-2}\right)$ and, for every positive $\varepsilon$, there are arbitrary large values of $x$ such that $\Psi(x) \geq x^{-2-\varepsilon}$ (hence, in particular, to all nonincreasing functions $\Psi: \mathbf{R}_{>0} \rightarrow \mathbf{R}_{>0}$ such that $\Psi(x)=o\left(x^{-2}\right)$ and the sum $\sum_{x \geq 1} x \Psi(x)$ diverges). This is contained in our Theorem 1.

The combination of Theorems B1 and 1 provides a satisfactory answer to Problem 1, except that $x \mapsto x^{2} \Psi(x)$ is assumed to be non-increasing in Theorem B1. However, by combining the strategy developed in [2] with the arguments used in the proof of Theorem 1 , we are able to remove this assumption. This is contained in our Theorem 3. Thus, we give a complete answer to Problem 1 and strengthen Theorem J, see Theorem 4 below.

One may, however, wish to strengthen Theorem $\mathrm{J}$ in another direction, that is, by relaxing the hypothesis on the function $\Psi$, which is assumed to be non-increasing. This assumption is needed to avoid the following situation. For a number $\xi$ in $\operatorname{Exact}(\Psi)$, there are rational numbers $p / q$ with arbitrarily large denominators such that

$$
\left|\xi-\frac{p}{q}\right|<\Psi(q) .
$$

Furthermore, by definition of the set $\operatorname{Exact}(\Psi)$, for every positive real number $\varepsilon$ and every positive integer $d$, we have

$$
\left|\xi-\frac{p}{q}\right|=\left|\xi-\frac{d p}{d q}\right|>(1-\varepsilon) \Psi(d q),
$$

if $q$ is sufficiently large in terms of $\varepsilon$. This gives a contradiction with $(1.2)$ when $(1-\varepsilon) \Psi(d q)$ exceeds $\Psi(q)$. Clearly, this situation cannot happen when $\Psi$ is non-increasing.

A second purpose of the present paper is to investigate whether Theorem $\mathrm{J}$ extends to non-monotonic functions $\Psi$. As far as we are aware, Problem 2 has not been studied yet.

Problem 2. Let $\Psi: \mathbf{R}_{>0} \rightarrow \mathbf{R}_{>0}$ be a function satisfying $\Psi(x)=o\left(x^{-2}\right)$. Is the set Exact $(\Psi)$ non-empty? To compute the Hausdorff dimension of $\operatorname{Exact}(\Psi)$.

Let us note that the study of $\operatorname{Exact}\left(x \mapsto c x^{-2}\right)$ for a positive real number $c$ amounts to the study of the Lagrange spectrum, see $[15,4,3,16]$. About this, we just mention that, for every positive $c$, the Hausdorff dimension of $\operatorname{Exact}\left(x \mapsto c x^{-2}\right)$ is strictly smaller than 
one (and this set can even be empty; this is the case for many values of $c$, for instance for every $c$ in $\left.\left(\frac{1}{\sqrt{13}}, \frac{1}{\sqrt{12}}\right)\right)$. This justifies the hypothesis $\Psi(x)=o\left(x^{-2}\right)$ of our main results.

Theorems 1, 2 and 3 give a partial answer to Problem 2. They are proved in Section 4, while Section 3 gathers auxiliary lemmas. Theorem 4, which is an immediate consequence of Theorems 1 and 3, gives a complete answer to Problem 1.

\section{Statements}

Motivated by a theorem of Duffin and Schaeffer [7], see Corollary 1 on page 27 of [9], for a function $\Psi: \mathbf{R}_{>0} \rightarrow \mathbf{R}_{>0}$, we say that $\Psi$ satisfies assumption (*) if

The function $\Psi$ satisfies $\Psi(x)=o\left(x^{-2}\right)$ and there exist real numbers $c, \tilde{c}$ and $n_{0}$ with $1 \leq \tilde{c}<4$ such that, if the positive integers $m, n$ satisfy $m>n \geq n_{0}$, then $\Psi(m) m^{c} \leq$ $\tilde{c} \Psi(n) n^{c}$.

We emphasize that the real number $c$ occurring in $(*)$ may be negative.

Our main result is a first step towards the resolution of Problem 2.

Theorem 1. Let $\Psi: \mathbf{R}_{>0} \rightarrow \mathbf{R}_{>0}$ be a function satisfying assumption (*). Suppose that, for every positive $\varepsilon$, there are infinitely many positive integers $n$ such that $\Psi(n)>n^{-2-\varepsilon}$. Then, the set $\operatorname{Exact}(\Psi)$ has full Hausdorff dimension.

By (1.1), for any given positive real number $\varepsilon$, the Hausdorff dimension of the set $\mathcal{K}\left(x \mapsto x^{-2-\varepsilon}\right)$ is equal to $2 /(2+\varepsilon)$. This explains the latter assumption on the function $\Psi$ in Theorem 1.

The proof of Theorem 1 rests on an idea from [16], which was also used in [18]. We construct a large subset of Exact $(\Psi)$ by suitably modifying sets of continued fractions with bounded partial quotients and arbitrarily large (albeit less than 1) Hausdorff dimension.

With the same method as for the proof of Theorem 1, we are able to give a partial answer to Problem 2 for every function $\Psi: \mathbf{R}_{>0} \rightarrow \mathbf{R}_{>0}$ satisfying assumption (*).

Theorem 2. Let $\Psi: \mathbf{R}_{>0} \rightarrow \mathbf{R}_{>0}$ be a function satisfying assumption (*). Then, the set $\operatorname{Exact}(\Psi)$ is uncountable.

In the course of the proof of Theorem 1, it is apparent that Problem 2 is connected with a well-known conjecture of Zaremba claiming that there exists a positive integer $M$ such that, for every integer $q \geq 2$, there is a positive integer $p$ coprime with $q$ and such that the partial quotients of the rational number $p / q$ are all less than $M$.

A suitable combination of the strategy developed in [2] with the arguments used in the proof of Theorem 1 allows us to extend Theorem B1 as follows.

Theorem 3. Let $\Psi: \mathbf{R}_{>0} \rightarrow \mathbf{R}_{>0}$ be a function satisfying assumption ( $*$ ). If $\lambda$ denotes the lower order at infinity of the function $1 / \Psi$, then

$$
\operatorname{dim} \operatorname{Exact}(\Psi)=\operatorname{dim} \mathcal{K}(\Psi)=\frac{2}{\lambda}
$$

The next theorem directly follows from Theorems 1 and 3, since every non-increasing function $\Psi$ with $\Psi(x)=o\left(x^{-2}\right)$ satisfies assumption $(*)$. 
Theorem 4. Let $\Psi: \mathbf{R}_{>0} \rightarrow \mathbf{R}_{>0}$ be a non-increasing function satisfying $\Psi(x)=o\left(x^{-2}\right)$. If $\lambda$ denotes the lower order at infinity of the function $1 / \Psi$, then

$$
\operatorname{dim} \operatorname{Exact}(\Psi)=\operatorname{dim} \mathcal{K}(\Psi)=\frac{2}{\lambda}
$$

Theorem 4 gives a complete answer to Problem 1.

\section{Auxiliary lemmas}

The key auxiliary lemma for the proof of Theorem 1 relates the Hausdorff dimension of a set and that of its image under an Hölderian map. We reproduce below Proposition 2.3 from [8].

Lemma 1. Let $F$ be a subset of $\mathbf{R}$. Let $f: F \rightarrow \mathbf{R}$ be a map for which there exist $c>0$ and $\alpha$ with $0<\alpha \leq 1$ such that

$$
|f(x)-f(y)| \leq c|x-y|^{\alpha}, \quad \text { for all } x, y \text { in } F .
$$

Then we have

$$
\operatorname{dim} F \geq \alpha \operatorname{dim} f(F) .
$$

For positive integers $a_{1}, \ldots, a_{n}$, we recall that the continuant $K\left(a_{1}, \ldots, a_{n}\right)$ denotes the denominator of the rational number $\left[0 ; a_{1}, \ldots, a_{n}\right]$. Next lemma is used in the proofs of Theorems 1 and 4 .

Lemma 2. Let $N$ be a positive integer. For any $\delta>0$, there is $K_{0}=K_{0}(\delta)>0$ such that, for any positive integers $a_{1}, \ldots, a_{n}$ such that $K\left(a_{1}, \ldots, a_{n}\right)<N / K_{0}$, the interval $(N /(1+\delta), N)$ contains at least one integer $q$ of the form

$$
q=K\left(a_{1}, \ldots, a_{n}, a_{n+1}, \ldots, a_{n+m}\right), \text { with } a_{n+1}, \ldots, a_{n+m} \in\{1,2\} .
$$

Proof. We will choose two large positive integers $r, s$, and take $m=r+s, a_{n+j}=1$ for $1 \leq j \leq r$ and $a_{n+j}=2$ for $r+1 \leq j \leq r+s=m$. Let $q_{k}=K\left(a_{1}, a_{2}, \ldots, a_{k}\right)$, for $1 \leq k \leq n+m$.

We have $q_{k+1}=q_{k}+q_{k-1}$, for $n \leq k \leq n+r-1$, and so $q_{n+j}=F_{j+1} q_{n}+F_{j} q_{n-1}$, for $0 \leq j \leq r$, where

$$
F_{j}=\frac{1}{\sqrt{5}}\left(\left(\frac{1+\sqrt{5}}{2}\right)^{j}-\left(\frac{1-\sqrt{5}}{2}\right)^{j}\right)=\frac{1+o(1)}{\sqrt{5}}\left(\frac{1+\sqrt{5}}{2}\right)^{j}
$$

is the $j$-th term of Fibonacci's sequence, for $j \geq 1$. So $q_{n+j}=(1+o(1)) c\left(\frac{1+\sqrt{5}}{2}\right)^{j} q_{n}$ for large $j$, where $c=\frac{1+\sqrt{5}}{2}+\frac{q_{n-1}}{q_{n}}$. On the other hand, we have $q_{k+1}=2 q_{k}+q_{k-1}$ for $n+r \leq k \leq n+m-1$, and so $q_{n+r+j}=u_{j+1} q_{n+r}+u_{j} q_{n+r-1}$, where $\left(u_{j}\right)_{j \geq 0}$ is the sequence given by $u_{0}=0, u_{1}=1$ and $u_{k+2}=2 u_{k+1}+u_{k}$, for $k \geq 0$. Since

$$
u_{k}=\frac{1}{2 \sqrt{2}}\left((1+\sqrt{2})^{k}-(1-\sqrt{2})^{k}\right)=\frac{1+o(1)}{2 \sqrt{2}}(1+\sqrt{2})^{k}, \quad \text { for } k \geq 0,
$$


we get

$$
\begin{aligned}
q_{n+r+j} & =\frac{1+o(1)}{2 \sqrt{2}}\left((1+\sqrt{2}) q_{n+r}+q_{n+r-1}\right)(1+\sqrt{2})^{j} \\
& =\frac{1+o(1)}{2 \sqrt{2}}\left(1+\sqrt{2}+\frac{\sqrt{5}-1}{2}\right) c(1+\sqrt{2})^{j}\left(\frac{1+\sqrt{5}}{2}\right)^{r} q_{n} \\
& =(1+o(1)) \frac{4+\sqrt{10}+\sqrt{2}}{8} c(1+\sqrt{2})^{j}\left(\frac{1+\sqrt{5}}{2}\right)^{r} q_{n}
\end{aligned}
$$

provided that $j$ and $r$ are large.

Since $\log (1+\sqrt{2}) / \log \left(\frac{1+\sqrt{5}}{2}\right)$ is irrational, the statement of the Lemma follows (by taking logarithms) from the elementary fact below:

Given $\alpha, \beta>0$ such that $\alpha / \beta$ is irrational, $\varepsilon>0$ and $r>0$, there is $x_{0}>0$ such that, for every $x \in \mathbf{R}, x \geq x_{0}$, there are positive integers $m, n \geq r$ such that $|m \alpha+n \beta-x|<\varepsilon$.

Under the notation of Lemma 2, with positive integers $a_{1}, \ldots, a_{n}$ and a positive real $\delta$, we associate integers $a_{n+1}, \ldots, a_{n+m}$ in $\{1,2\}$ in such a way that

$$
N /(1+\delta)<K\left(a_{1}, \ldots, a_{n}, a_{n+1}, \ldots, a_{n+m}\right)<N .
$$

There may be multiple choices, but we select one of them and define in this way a map $\Theta$.

Remark. Replacing $(N /(1+\delta), N)$ by $(N-\delta(N), N)$ for a function $\delta$ satisfying $\delta(N)=$ $o(N)$ would allow us to weaken the assumption $(*)$ in Theorems 1 and 4 . We may even hope that there exist positive integers $M$ and $Q$ such that, under the assumption of Lemma 2 , for every sufficiently large integer $q$, at least one of the integers $q, q+1, \ldots, q+Q$ is of the form

$$
K\left(a_{1}, \ldots, a_{n}, a_{n+1}, \ldots, a_{n+m}\right), \quad a_{n+1}, \ldots, a_{n+m} \in\{1,2, \ldots, M\}
$$

We cannot exclude that every sufficiently large integer can be written under this form.

We also need the following elementary facts about continued fractions:

Lemma 3. i) Given an irrational real number $\alpha=\left[a_{0} ; a_{1}, a_{2}, \ldots\right]$, the sequence of its convergents $p_{n} / q_{n}=\left[a_{0} ; a_{1}, a_{2}, \ldots, a_{n}\right]$ (where $\left.q_{n}=K\left(a_{1}, a_{2}, \ldots, a_{n}\right)\right)$ satisfies

$$
\frac{1}{\left(a_{n+1}+2\right) q_{n}^{2}}<\left|\alpha-\frac{p_{n}}{q_{n}}\right|<\frac{1}{a_{n+1} q_{n}^{2}} \leq \frac{1}{q_{n}^{2}} .
$$

ii) For any finite sequences $\left(a_{1}, a_{2}, \ldots, a_{m}\right),\left(b_{1}, b_{2}, \ldots, b_{n}\right)$ of positive integers, we have

$$
\begin{aligned}
K\left(a_{1}, a_{2}, \ldots, a_{m}\right) K\left(b_{1}, b_{2}, \ldots, b_{n}\right) & \leq K\left(a_{1}, a_{2}, \ldots, a_{m}, b_{1}, b_{2}, \ldots, b_{n}\right) \\
& <2 K\left(a_{1}, a_{2}, \ldots, a_{m}\right) K\left(b_{1}, b_{2}, \ldots, b_{n}\right) .
\end{aligned}
$$

iii) If $\alpha=\left[a_{0} ; a_{1}, a_{2}, \ldots\right], \alpha^{\prime}=\left[a_{0}^{\prime} ; a_{1}^{\prime}, a_{2}^{\prime}, \ldots\right]$ are such that $a_{j}=a_{j}^{\prime}, 0 \leq j \leq n$ and $a_{n+1} \neq a_{n+1}^{\prime}$, then $\left|\alpha-\alpha^{\prime}\right|>\frac{1}{\left(a_{n+1}+1\right)\left(a_{n+1}^{\prime}+1\right)\left(\max \left\{a_{n+2}, a_{n+2}^{\prime}\right\}+1\right) q_{n}^{2}}$.

In particular, if $a_{n+1}, a_{n+1}^{\prime}, a_{n+2}, a_{n+2}^{\prime} \leq m$ then $\left|\alpha-\alpha^{\prime}\right|>\frac{1}{(m+1)^{3} q_{n}^{2}}$. 
Proof. i) and ii) are well-known facts. In order to prove iii), we use that

$$
\alpha=\frac{\alpha_{n+1} p_{n}+p_{n-1}}{\alpha_{n+1} q_{n}+q_{n-1}}, \quad \alpha^{\prime}=\frac{\alpha_{n+1}^{\prime} p_{n}+p_{n-1}}{\alpha_{n+1}^{\prime} q_{n}+q_{n-1}}
$$

where

$$
\alpha_{n+1}=\left[a_{n+1} ; a_{n+2}, a_{n+3}, \ldots\right], \alpha_{n+1}^{\prime}=\left[a_{n+1}^{\prime} ; a_{n+2}^{\prime}, a_{n+3}^{\prime}, \ldots\right] .
$$

So we have

$$
\begin{aligned}
\left|\alpha-\alpha^{\prime}\right|=\left|\frac{\left(p_{n} q_{n-1}-p_{n-1} q_{n}\right)\left(\alpha_{n+1}-\alpha_{n+1}^{\prime}\right)}{\left(\alpha_{n+1} q_{n}+q_{n-1}\right)\left(\alpha_{n+1}^{\prime} q_{n}+q_{n-1}\right)}\right| & =\left|\frac{\alpha_{n+1}-\alpha_{n+1}^{\prime}}{\left(\alpha_{n+1} q_{n}+q_{n-1}\right)\left(\alpha_{n+1}^{\prime} q_{n}+q_{n-1}\right)}\right| \\
& >\left|\frac{\alpha_{n+1}-\alpha_{n+1}^{\prime}}{\left(a_{n+1}+1\right)\left(a_{n+1}^{\prime}+1\right) q_{n}^{2}}\right| .
\end{aligned}
$$

On the other hand, assuming, without loss of generality, that $a_{n+1}>a_{n+1}^{\prime}$, we have $\alpha_{n+1}^{\prime}<a_{n+1}^{\prime}+1 \leq a_{n+1}$ and $\alpha_{n+1}>a_{n+1}+1 /\left(a_{n+2}+1\right)$, and so $\left|\alpha_{n+1}-\alpha_{n+1}^{\prime}\right|=$ $\alpha_{n+1}-\alpha_{n+1}^{\prime}>1 /\left(a_{n+2}+1\right) \geq 1 /\left(\max \left\{a_{n+2}, a_{n+2}^{\prime}\right\}+1\right)$, which implies the result.

\section{Proofs}

Proof of Theorem 1.

Let $\Psi$ be a function as in the statement of Theorem 1 (in particular $\Psi$ satisfies assumption $(*)$ for some real numbers $n_{0}, c$ and $\left.\tilde{c}\right)$. We use a method applied successfully in [16] (see also [18]), that consists in slightly perturbating continued fractions with bounded coefficients to construct many real numbers in $\operatorname{Exact}(\Psi)$.

For a given integer $m \geq 8$, let $C_{m}$ be the set of real numbers in $(0,1)$ whose partial quotients are at most equal to $m$. Jarník [10] established that $\operatorname{dim} C_{m}>1-1 /(m \log 2)$. We construct a suitable map $h_{m}$ from $C_{m}$ to $\operatorname{Exact}(\Psi)$ such that, for any $\alpha$ with $0<\alpha<1$, we have $\left|\xi-\xi^{\prime}\right|=O\left(\left|h_{m}(\xi)-h_{m}\left(\xi^{\prime}\right)\right|^{\alpha}\right)$. By Lemma 1 and Jarník's aforementioned result, this implies that

$$
\operatorname{dim} \operatorname{Exact}(\Psi) \geq \operatorname{dim} C_{m}>1-\frac{1}{m \log 2}
$$

for every integer $m \geq 8$, and so $\operatorname{dim} \operatorname{Exact}(\Psi)=1$, which is the conclusion of Theorem 1 .

Let $\delta$ be a positive real number such that

$$
(1+\delta)^{|c|+2} \leq \frac{2}{\sqrt{\tilde{c}}}
$$

Let us, from now on, fix an integer $m \geq 8$. In all what follows, $\lceil x\rceil$ denotes the smallest integer greater than or equal to $x$.

We construct inductively a rapidly increasing sequence $\left(n_{k}\right)_{k \geq 1}$ of integers satisfying $n_{1}=\left\lceil(m+1) K_{0}(\delta)\right\rceil$, where $K_{0}(\delta)$ is the constant given by Lemma 2 ,

$$
n_{k}>n_{k-1}^{3}, \quad(k \geq 2),
$$




$$
\Psi\left(n_{k}\right)>\frac{1}{n_{k}^{2+1 / k}}, \quad(k \geq 2),
$$

and

$$
r^{2} \Psi(r)<n_{k}^{2} \Psi\left(n_{k}\right) \quad \text { for all } r>n_{k}, \quad(k \geq 2) .
$$

In order to do this, for each $k \geq 2$, we define $\tilde{n}_{k}$ as the smallest positive integer $n$ such that $n>n_{k-1}^{3}$ and $\Psi(n)>n^{-2-1 / k}$ (which is possible since for every positive $\varepsilon$, there are infinitely many positive integers $n$ such that $\Psi(n)>n^{-2-\varepsilon}$ ), and then define $n_{k}$ as

$$
n_{k}:=\max \left\{r \geq \tilde{n}_{k}: r^{2} \Psi(r) \geq \tilde{n}_{k}^{2} \Psi\left(\tilde{n}_{k}\right)\right\},
$$

which is possible since $\Psi(x)=o\left(x^{-2}\right)$.

Notice that $n_{k}^{2} \Psi\left(n_{k}\right) \geq \tilde{n}_{k}^{2} \Psi\left(\tilde{n}_{k}\right)>\tilde{n}_{k}^{-1 / k} \geq n_{k}{ }^{-1 / k}$, so $\Psi\left(n_{k}\right)>n_{k}^{-2-1 / k}$ and, for every $r>n_{k}$, we have $r^{2} \Psi(r)<\tilde{n}_{k}^{2} \Psi\left(\tilde{n}_{k}\right) \leq n_{k}^{2} \Psi\left(n_{k}\right)$.

Now we describe the map $h_{m}$ from $C_{m}$ to $\operatorname{Exact}(\Psi)$. Let $\xi=\left[0 ; a_{1}, a_{2}, \ldots\right]$ be in $C_{m}$. We will have $h_{m}(\xi)=\left[0 ; b_{1}, b_{2}, b_{3}, \ldots\right]$, where the continued fraction $\left[0 ; b_{1}, b_{2}, b_{3}, \ldots\right]$ is obtained from the continued fraction $\left[0 ; a_{1}, a_{2}, a_{3}, \ldots\right]$ of $\xi$ by conveniently inserting in it a sequence of finite blocks of coefficients, in order to create, for each positive integer $k$, a convergent $p_{m_{k}} / q_{m_{k}}$ of $h_{m}(\xi)$ with

$$
n_{k} /(1+\delta)<q_{m_{k}} \leq n_{k}
$$

Each of these blocks will end by a term of the type $b_{m_{k}+1}=\left\lceil\frac{1}{q_{m_{k}}^{2} \Psi\left(q_{m_{k}}\right)}\right\rceil$, which makes $\left|h_{m}(\xi)-p_{m_{k}} / q_{m_{k}}\right|$ very close to $\Psi\left(q_{m_{k}}\right)$.

More precisely, we will put

$$
\begin{gathered}
h_{m}(\xi)=\left[0 ; b_{1}, b_{2}, b_{3}, \ldots\right]=\left[0 ; a_{1}, a_{2}, \ldots, a_{r_{1}}, c_{1}^{(1)}, c_{2}^{(1)}, \ldots \ldots, c_{s_{1}}^{(1)}, c_{s_{1}+1}^{(1)}, a_{r_{1}+1},\right. \\
\left.a_{r_{1}+2}, \ldots, a_{r_{2}}, c_{1}^{(2)}, c_{2}^{(2)}, \ldots, c_{s_{2}}^{(2)}, c_{s_{2}+1}^{(2)}, a_{r_{2}+1}, a_{r_{2}+2}, \ldots\right]
\end{gathered}
$$

where, for each $j \geq 1, r_{j}$ is the smallest $r$ such that

$$
\begin{gathered}
K\left(a_{1}, a_{2}, \ldots, a_{r_{1}}, c_{1}^{(1)}, c_{2}^{(1)}, \ldots, c_{s_{1}}^{(1)}, c_{s_{1}+1}^{(1)}, a_{r_{1}+1}, a_{r_{1}+2}, \ldots, a_{r_{j-1}+1}, a_{r_{j-1}+2}, \ldots, a_{r}\right) \\
>\frac{n_{j}}{(m+1) K_{0}(\delta)} .
\end{gathered}
$$

By the minimality of $r_{j}$ and since $a_{r_{j}} \leq m$, it follows from Lemma 3 that

$$
\begin{gathered}
K\left(a_{1}, a_{2}, \ldots, a_{r_{1}}, c_{1}^{(1)}, c_{2}^{(1)}, \ldots, c_{s_{1}}^{(1)}, c_{s_{1}+1}^{(1)}, a_{r_{1}+1}, a_{r_{1}+2}, \ldots, a_{r_{j-1}+1}, a_{r_{j-1}+2}, \ldots, a_{r_{j}}\right) \\
<n_{j} / K_{0}(\delta) .
\end{gathered}
$$

Now, we use Lemma 2 and the map $\Theta$ defined after the proof that lemma to find integers $c_{1}^{(j)}, c_{2}^{(j)}, \ldots, c_{s_{j}}^{(j)}$ in $\{1,2\}$ such that $n_{j} /(1+\delta)<q_{m_{j}} \leq n_{j}$, where $m_{j}:=r_{j}+s_{j}+$ $\sum_{1 \leq i<j}\left(s_{i}+1\right)$. 
Then we take $c_{s_{j}+1}^{(j)}=\left\lceil\frac{1}{q_{m_{j}}^{2} \Psi\left(q_{m_{j}}\right)}\right\rceil$, and we continue this construction for each $j$.

Since $\Psi(x)=o\left(x^{-2}\right)$ and the only coefficients of the continued fraction $\left[0 ; b_{1}, b_{2}, b_{3}, \ldots\right]$ of $h_{m}(\xi)$ which can be larger than $m$ are the coefficients $c_{s_{j}+1}^{(j)}$, the inequality $\left|h_{m}(\xi)-p / q\right|<$ $\Psi(q)$, with $q$ large, implies that $p / q=p_{m_{k}} / q_{m_{k}}$ for some $k$.

Now, since we have, by Lemma 3 ,

$$
\begin{aligned}
\frac{\sqrt{\tilde{c}}}{2} \Psi\left(q_{m_{k}}\right)<\frac{1}{\left(c_{s_{k}+1}^{(k)}+2\right) q_{m_{k}}^{2}} & <\left|h_{m}(\xi)-\frac{p_{m_{k}}}{q_{m_{k}}}\right| \\
& <\frac{1}{c_{s_{k}+1}^{(k)} q_{m_{k}}^{2}}=\frac{1}{\left\lceil\frac{1}{q_{m_{k}}^{2} \Psi\left(q_{m_{k}}\right)}\right\rceil q_{m_{k}}^{2}} \leq \Psi\left(q_{m_{k}}\right),
\end{aligned}
$$

for large $k$, it is enough to show that, for $k$ large, the approximations $\frac{d p_{m_{k}}}{d q_{m_{k}}}$ of $h_{m}(\xi)$ for integers $d \geq 2$ do not satisfy $\left|h_{m}(\xi)-\frac{d p_{m_{k}}}{d q_{m_{k}}}\right|<\Psi\left(d q_{m_{k}}\right)$ in order to conclude that $h_{m}(\xi)$ is in $\operatorname{Exact}(\Psi)$.

Since $2 q_{m_{k}}$ exceeds $n_{k}$, we infer from (4.4) that, for every integer $d \geq 2$, we have

$$
\left(d q_{m_{k}}\right)^{2} \Psi\left(d q_{m_{k}}\right)<n_{k}^{2} \Psi\left(n_{k}\right)
$$

thus, using (4.1), (4.5) and the assumption $(*)$, we get

$$
\begin{aligned}
\Psi\left(d q_{m_{k}}\right)<\frac{1}{4}\left(\frac{n_{k}}{q_{m_{k}}}\right)^{2} \Psi\left(n_{k}\right) & \leq \frac{(1+\delta)^{2}}{4} \tilde{c} \Psi\left(q_{m_{k}}\right)\left(\frac{q_{m_{k}}}{n_{k}}\right)^{c} \\
& \leq \frac{\tilde{c}}{4}(1+\delta)^{|c|+2} \Psi\left(q_{m_{k}}\right) \leq \frac{\sqrt{\tilde{c}}}{2} \Psi\left(q_{m_{k}}\right) .
\end{aligned}
$$

So, for large $k$, we get from (4.6) that

$$
\Psi\left(d q_{m_{k}}\right)<\frac{\sqrt{\tilde{c}}}{2} \Psi\left(q_{m_{k}}\right)<\frac{1}{\left(c_{s_{k}+1}^{(k)}+2\right) q_{m_{k}}^{2}}<\left|h_{m}(\xi)-\frac{p_{m_{k}}}{q_{m_{k}}}\right|
$$

which concludes the proof that $h_{m}(\xi)$ is in $\operatorname{Exact}(\Psi)$.

We will check now that, for any $\xi, \xi^{\prime}$ in $C_{m}$ and any $\alpha$ with $0<\alpha<1$, we have

$$
\left|\xi-\xi^{\prime}\right|=O\left(\left|h_{m}(\xi)-h_{m}\left(\xi^{\prime}\right)\right|^{\alpha}\right)
$$

Let $\xi=\left[0 ; a_{1}, a_{2}, a_{3}, \ldots\right]$ and $\xi^{\prime}=\left[0 ; a_{1}^{\prime}, a_{2}^{\prime}, a_{3}^{\prime}, \ldots\right]$ be in $C_{m}$, and let $\tilde{n}$ be the least positive integer $i$ such that $a_{i} \neq a_{i}^{\prime}$.

We have

$$
\begin{gathered}
h_{m}(\xi)=\left[0 ; b_{1}, b_{2}, b_{3}, \ldots\right]=\left[0 ; a_{1}, a_{2}, \ldots, a_{r_{1}}, c_{1}^{(1)}, c_{2}^{(1)}, \ldots, c_{s_{1}}^{(1)}, c_{s_{1}+1}^{(1)}, a_{r_{1}+1},\right. \\
\left.a_{r_{1}+2}, \ldots, a_{r_{2}}, c_{1}^{(2)}, c_{2}^{(2)}, \ldots, c_{s_{2}}^{(2)}, c_{s_{2}+1}^{(2)}, a_{r_{2}+1}, a_{r_{2}+2}, \ldots\right]
\end{gathered}
$$


and

$$
\begin{gathered}
h_{m}\left(\xi^{\prime}\right)=\left[0 ; b_{1}^{\prime}, b_{2}^{\prime}, b_{3}^{\prime}, \ldots\right]=\left[0 ; a_{1}^{\prime}, a_{2}^{\prime}, \ldots, a_{r_{1}^{\prime}}^{\prime}, c_{1}^{\prime(1)}, c_{2}^{\prime(1)}, \ldots, c_{s_{1}^{\prime}}^{(1)}, c_{s_{1}^{\prime}+1}^{\prime(1)}, a_{r_{1}^{\prime}+1}^{\prime},\right. \\
\left.a_{r_{1}^{\prime}+2}^{\prime}, \ldots, a_{r_{2}^{\prime}}^{\prime}, c_{1}^{\prime(2)}, c_{2}^{\prime(2)}, \ldots, c_{s_{2}^{\prime}}^{(2)}, c_{s_{2}^{\prime}+1}^{(2)}, a_{r_{2}^{\prime}+1}^{\prime}, a_{r_{2}^{\prime}+2}^{\prime}, \ldots\right] .
\end{gathered}
$$

Let $j$ be the smallest index such that $\tilde{n} \leq r_{j+1}$. We have, for every $i \leq j, a_{k}^{\prime}=a_{k}$ for all $k \leq r_{i}, r_{i}=r_{i}^{\prime}, s_{i}=s_{i}^{\prime}$ for all $i \leq j$ and $c_{r}^{(i)}={c^{\prime}}_{r}^{(i)}$, for all $i \leq j, r \leq s_{i}+1=s_{i}^{\prime}+1$ (recall that we have used the map $\Theta$ in the construction of $h_{m}$ ). This means that $b_{i}=b_{i}^{\prime}$ for all $i \leq m_{j}+\tilde{n}-r_{j}$, where $m_{j}=r_{j}+s_{j}+\sum_{1 \leq i<j}\left(s_{i}+1\right)$, and we have $b_{m_{j}+1+\tilde{n}-r_{j}}=a_{\tilde{n}} \neq$ $a_{\tilde{n}}^{\prime}=b_{m_{j}+1+\tilde{n}-r_{j}}^{\prime}$.

Let $k=1, \ldots, j$. We infer from (4.1), (4.3), (4.5) and $\Psi\left(q_{m_{k}}\right) q_{m_{k}}^{c} \geq \Psi\left(n_{k}\right) n_{k}^{c} / \tilde{c}$ that

$$
\Psi\left(q_{m_{k}}\right) \geq \frac{1}{\tilde{c}}(1+\delta)^{-|c|} \Psi\left(n_{k}\right) \geq \frac{\Psi\left(n_{k}\right)}{2 \sqrt{\tilde{c}}}>\frac{1}{2 \sqrt{\tilde{c}} n_{k}^{2+1 / k}} \geq \frac{1}{6 q_{m_{k}}^{2+1 / k}}
$$

The latter inequality holds since $n_{k}^{-2-1 / k}>(1+\delta)^{-2-1 / k} q_{m_{k}}^{-2-1 / k} \geq(1+\delta)^{-3} q_{m_{k}}^{-2-1 / k}$, and $(1+\delta)^{3} \leq(2 / \sqrt{\tilde{c}})^{3 / 2}<3 / \sqrt{\tilde{c}}$.

In particular, we have

$$
c_{s_{k}+1}^{(k)}=\left\lceil\frac{1}{q_{m_{k}}^{2} \Psi\left(q_{m_{k}}\right)}\right\rceil<6 q_{m_{k}}^{1 / k}+1
$$

and so

$$
q_{m_{k}+1}<\left(6 q_{m_{k}}^{1 / k}+2\right) q_{m_{k}} \leq 8 q_{m_{k}}^{1+1 / k} .
$$

Furthermore, from the construction of $h_{m}(\xi)$, we get that $q_{m_{k}} / q_{m_{k}-s_{k}}<(m+1) K_{0}(\delta)$.

Moreover, using Lemma 3, item ii), we can conclude that

$$
\begin{aligned}
q_{m_{k}+1} & <8(m+1) K_{0}(\delta) q_{m_{k}}^{1 / k} q_{m_{k}-s_{k}} \\
& <8(m+1) K_{0}(\delta) n_{k}^{1 / k} q_{m_{k}-s_{k}} \\
& <16(m+1) K_{0}(\delta) n_{k}^{1 / k} K\left(a_{r_{k-1}+1}, a_{r_{k-1}+2}, \ldots, a_{r_{k}}\right) q_{m_{k-1}+1}
\end{aligned}
$$

for $k \leq j$.

Finally, we have

$$
q_{m_{j}+\tilde{n}-r_{j}}<2 K\left(a_{r_{j}+1}, a_{r_{j}+2}, \ldots, a_{\tilde{n}-1}\right) q_{m_{j}+1},
$$

with the convention that $K\left(a_{r_{j}+1}, a_{r_{j}}\right)=1$.

Therefore, setting $r_{0}=0$, we deduce from the preceding estimates that $q_{m_{j}+\tilde{n}-r_{j}}$ is smaller than

$$
\begin{aligned}
& \left(16(m+1) K_{0}(\delta)\right)^{j} \Pi_{k=1}^{j}\left(n_{k}^{1 / k} \cdot K\left(a_{r_{k-1}+1}, a_{r_{k-1}+2}, \ldots, a_{r_{k}}\right)\right) \cdot K\left(a_{r_{j}+1}, a_{r_{j}+2}, \ldots, a_{\tilde{n}-1}\right) \\
& \quad \leq\left(16(m+1) K_{0}(\delta)\right)^{j} \Pi_{k=1}^{j} n_{k}^{1 / k} \cdot K\left(a_{1}, a_{2}, \ldots, a_{\tilde{n}-1}\right) .
\end{aligned}
$$


Since $\Pi_{k=1}^{j} n_{k}^{1 / k}=\exp \left(\sum_{k=1}^{j}\left(\log n_{k}\right) / k\right)$, and $\log n_{k-1} \leq\left(\log n_{k}\right) / 3$, for $k \leq j$, we get $\Pi_{k=1}^{j} n_{k}^{1 / k} \leq n_{j}^{3 /(2 j)}$. Using that $n_{j} \geq\left((m+1) K_{0}(\delta)\right)^{3^{j-1}}$, we deduce that $\left(16(m+1) K_{0}(\delta)\right)^{j}=\left(n_{j}^{1 / j}\right)^{o(1)}$, and so

$$
\left(16(m+1) K_{0}(\delta)\right)^{j} \Pi_{k=1}^{j} n_{k}^{1 / k} \leq n_{j}^{(3+o(1)) / 2 j}=q_{m_{j}}^{(3+o(1)) / 2 j}=q_{m_{j}}^{o(1)}=q_{m_{j}+\tilde{n}-r_{j}}^{o(1)} .
$$

Summarizing, we have $q_{m_{j}+\tilde{n}-r_{j}}<q_{m_{j}+\tilde{n}-r_{j}}^{o(1)} \cdot K\left(a_{1}, a_{2}, \ldots, a_{\tilde{n}-1}\right)$, and so

$$
q_{m_{j}+\tilde{n}-r_{j}}^{1-o(1)}<K\left(a_{1}, a_{2}, \ldots, a_{\tilde{n}-1}\right) .
$$

From Lemma 3, item i), we have

$$
\left|\xi-\xi^{\prime}\right|<\frac{1}{K\left(a_{1}, a_{2}, \ldots, a_{\tilde{n}-1}\right)^{2}}<\frac{1}{q_{m_{j}+\tilde{n}-r_{j}}^{2(1-o(1))}}
$$

and, by Lemma 3, item iii),

$$
\left|h_{m}(\xi)-h_{m}\left(\xi^{\prime}\right)\right| \geq \frac{1}{(m+1)^{3} q_{m_{j}+\tilde{n}-r_{j}}^{2}}=\frac{1}{q_{m_{j}+\tilde{n}-r_{j}}^{2+o(1)}} .
$$

We then conclude that $|\alpha-\beta|<\left|h_{m}(\alpha)-h_{m}(\beta)\right|^{1-o(1)}$. This finishes the proof of Theorem 1 .

\section{Proof of Theorem 2.}

Let $\Psi$ be a function satisfying assumption $(*)$ for some real numbers $n_{0}$ and $c$. Let $N_{0}$ be such that $\Psi(n)<1 / n^{2}$ for $n \geq N_{0}$.

We construct inductively a rapidly increasing sequence $\left(\hat{n}_{k}\right)_{k \geq 1}$ of integers defined by $\hat{n}_{1}=\max \left\{N_{0},\left\lceil 3 K_{0}(\delta)\right\rceil\right\}$, where $K_{0}(\delta)$ is the constant given by Lemma 2 , and

$$
\hat{n}_{k+1}=\min \left\{n \text { positive integer }: n>\left\lceil 1 / \Psi\left(\hat{n}_{k}\right)\right\rceil \text { and } n^{2} \Psi(n)>r^{2} \Psi(r) \text {, for every } r>n\right\},
$$

for $k \geq 1$ (this is possible since $\Psi(x)=o\left(x^{-2}\right)$ ). We construct a continuous injective map $\hat{h}$ from the set $C_{2}$ of real numbers with partial quotients in $\{1,2\}$ to the set $\operatorname{Exact}(\Psi)$, which implies the result. Let $\xi=\left[0 ; a_{1}, a_{2}, \ldots\right]$ be in $C_{2}$ and write

$$
\begin{gathered}
\hat{h}(\xi)=\left[0 ; b_{1}, b_{2}, b_{3}, \ldots\right]=\left[0 ; a_{1}, a_{2}, \ldots, a_{r_{1}}, c_{1}^{(1)}, c_{2}^{(1)}, \ldots, c_{s_{1}}^{(1)}, c_{s_{1}+1}^{(1)}, a_{r_{1}+1},\right. \\
\left.a_{r_{1}+2}, \ldots, a_{r_{2}}, c_{1}^{(2)}, c_{2}^{(2)}, \ldots, c_{s_{2}}^{(2)}, c_{s_{2}+1}^{(2)}, a_{r_{2}+1}, a_{r_{2}+2}, \ldots\right]
\end{gathered}
$$

where, for each $j \geq 1$, the integer $r_{j}$ is the smallest $r$ such that

$$
\begin{gathered}
K\left(a_{1}, a_{2}, \ldots, a_{r_{1}}, c_{1}^{(1)}, c_{2}^{(1)}, \ldots, c_{s_{1}}^{(1)}, c_{s_{1}+1}^{(1)}, a_{r_{1}+1}, a_{r_{1}+2}, \ldots, a_{r_{j-1}+1}, a_{r_{j-1}+2}, \ldots, a_{r}\right) \\
>\frac{\hat{n}_{j}}{3 K_{0}(\delta)} .
\end{gathered}
$$


By the minimality of $r_{j}$ and since $a_{r_{j}} \leq 2$, we have

$$
\begin{gathered}
K\left(a_{1}, a_{2}, \ldots, a_{r_{1}}, c_{1}^{(1)}, c_{2}^{(1)}, \ldots, c_{s_{1}}^{(1)}, c_{s_{1}+1}^{(1)}, a_{r_{1}+1}, a_{r_{1}+2}, \ldots, a_{r_{j-1}+1}, a_{r_{j-1}+2}, \ldots, a_{r_{j}}\right) \\
<\hat{n}_{j} / K_{0}(\delta) .
\end{gathered}
$$

Now, we use Lemma 2 to find $c_{1}^{(j)}, c_{2}^{(j)}, \ldots, c_{s_{j}}^{(j)}$ in $\{1,2\}$ such that

$$
\hat{n}_{j} /(1+\delta)<q_{m_{j}} \leq \hat{n}_{j}
$$

where $m_{j}:=r_{j}+s_{j}+\sum_{1 \leq i<j}\left(s_{i}+1\right)$. Then we take $c_{s_{j}+1}^{(j)}=\left\lceil\frac{1}{q_{m_{j}}^{2} \Psi\left(q_{m_{j}}\right)}\right\rceil$, and we continue this construction for each $j$.

By the construction, the map $\hat{h}$ is clearly continuous and injective, and, as in the proof of Theorem 1, we can show that $\hat{h}(\xi)$ is in $\operatorname{Exact}(\Psi)$ for $\xi$ in $C_{2}$. This establishes Theorem 2 .

\section{Proof of Theorem 3.}

Let $\Psi$ be a function satisfying assumption $(*)$ for some real numbers $n_{0}$ and $c$, such that the function $1 / \Psi$ has lower order $\lambda$ at infinity. In view of Theorem 1 , we may assume without loss of generality that $\lambda>2$. A classical covering argument shows that the Hausdorff dimension of the set $\mathcal{K}(\Psi)$ (which contains $\operatorname{Exact}(\Psi)$ ) is at most equal to $2 / \lambda$. To prove that this is the exact value of the dimension is more difficult. In order to do this, we will combine the technique of the proofs of the previous theorems with ideas of [2]. We will assume from now on that $\lambda$ is finite.

Let $N_{0}$ be such that $\Psi(n)<1 / n^{2}$ for $n \geq N_{0}$. Let $m \geq 8$ be an integer. We construct inductively a rapidly increasing sequence $\left(\check{n}_{k}\right)_{k \geq 1}$ of integers defined by $\check{n}_{1}=$ $\max \left\{N_{0},\left\lceil(m+1) K_{0}(\delta)\right\rceil\right\}$, where $K_{0}(\delta)$ is the constant given by Lemma 2 ,

$$
\begin{array}{r}
\bar{n}_{k}=\min \left\{n \text { positive integer }: n>\max \left\{\left\lceil 1 / \Psi\left(\check{n}_{k}\right)\right\rceil, \check{n}_{k}^{k}\right\}\right. \\
\text { and } \left.\Psi(n)>n^{-\lambda-1 / k}\right\},
\end{array}
$$

and

$$
\check{n}_{k+1}:=\max \left\{r \geq \bar{n}_{k}: r^{2} \Psi(r) \geq \bar{n}_{k}^{2} \Psi\left(\bar{n}_{k}\right)\right\},
$$

for $k \geq 1$ (this is possible since $\Psi(x)=o\left(x^{-2}\right)$ ).

Let $\xi=\left[0 ; a_{1}, a_{2}, \ldots\right]$ be in $C_{m}$. We will construct continued fractions of the type

$$
\begin{gathered}
\xi=\left[0 ; b_{1}, b_{2}, b_{3}, \ldots\right]=\left[0 ; a_{1}, a_{2}, \ldots, a_{r_{1}}, c_{1}^{(1)}, c_{2}^{(1)}, \ldots, c_{s_{1}}^{(1)}, c_{s_{1}+1}^{(1)}, a_{r_{1}+1},\right. \\
\left.a_{r_{1}+2}, \ldots, a_{r_{2}}, c_{1}^{(2)}, c_{2}^{(2)}, \ldots, c_{s_{2}}^{(2)}, c_{s_{2}+1}^{(2)}, a_{r_{2}+1}, a_{r_{2}+2}, \ldots\right]
\end{gathered}
$$

where, for each $j \geq 1$, the integer $r_{j}$ is the smallest $r$ such that

$$
\begin{gathered}
K\left(a_{1}, a_{2}, \ldots, a_{r_{1}}, c_{1}^{(1)}, c_{2}^{(1)}, \ldots, c_{s_{1}}^{(1)}, c_{s_{1}+1}^{(1)}, a_{r_{1}+1}, a_{r_{1}+2}, \ldots, a_{r_{j-1}+1}, a_{r_{j-1}+2}, \ldots, a_{r}\right) \\
>\frac{\check{n}_{j}}{(m+1) K_{0}(\delta)} .
\end{gathered}
$$


By the minimality of $r_{j}$ and since $a_{r_{j}} \leq m$, we have

$$
\begin{gathered}
K\left(a_{1}, a_{2}, \ldots, a_{r_{1}}, c_{1}^{(1)}, c_{2}^{(1)}, \ldots, c_{s_{1}}^{(1)}, c_{s_{1}+1}^{(1)}, a_{r_{1}+1}, a_{r_{1}+2}, \ldots, a_{r_{j-1}+1}, a_{r_{j-1}+2}, \ldots, a_{r_{j}}\right) \\
<\check{n}_{j} / K_{0}(\delta) .
\end{gathered}
$$

Now, we use Lemma 2 to find $c_{1}^{(j)}, c_{2}^{(j)}, \ldots, c_{s_{j}}^{(j)}$ in $\{1,2\}$ such that

$$
\check{n}_{j} /(1+\delta)<q_{m_{j}} \leq \check{n}_{j}
$$

where $m_{j}:=r_{j}+s_{j}+\sum_{1 \leq i<j}\left(s_{i}+1\right)$. Then we take for $c_{s_{j}+1}^{(j)}$ an (arbitrary) integer between $\left\lceil\frac{1}{q_{m_{j}}^{2} \Psi\left(q_{m_{j}}\right)}\right\rceil$ and $\left\lceil\left(1+\frac{1}{j}\right) \frac{1}{q_{m_{j}}^{2} \Psi\left(q_{m_{j}}\right)}\right\rceil$, and we continue this construction for each $j$.

We can show as in the proof of Theorem 1 that any real number $\xi$ constructed in this way is in $\operatorname{Exact}(\Psi)$. The set $\hat{\mathcal{C}}$ of possible real numbers $\xi$ constructed in this way is a Cantor set whose Hausdorff dimension will be estimated below.

Let us recall the statement of Proposition 1 of [2] (which is Example 4.6 of [8]). Consider a decreasing sequence of sets $[0,1]=E_{0} \supset E_{1} \supset E_{2} \supset \ldots$ such that each $E_{k}$ is a finite disjoint union of closed intervals. Assume that for each $k \geq 1$, each interval of $E_{k-1}$ contains at least $\hat{m}_{k} \geq 2$ intervals of $E_{k}$ which are separated by gaps of size at least $\varepsilon_{k}$, where $0<\varepsilon_{k+1}<\varepsilon_{k}$. Then the Hausdorff dimension of the Cantor set $\mathcal{C}:=\cap_{k=0}^{\infty} E_{k}$ satisfies

$$
\operatorname{dim} \mathcal{C} \geq \liminf _{k \rightarrow+\infty} \frac{\log \left(\hat{m}_{1} \ldots \hat{m}_{k-1}\right)}{-\log \left(\hat{m}_{k} \varepsilon_{k}\right)}
$$

We will describe sets $\hat{E}_{k}$, which are disjoint unions of closed intervals, satisfying $\hat{\mathcal{C}}=$ $\cap_{k=0}^{\infty} \hat{E}_{k}$, which allow us to use the above proposition to estimate $\operatorname{dim} \hat{\mathcal{C}}$. In order to do this, we will describe, for each $\xi \in \hat{\mathcal{C}}$, and each $k \geq 1$, the component interval $\hat{I}_{k}(\xi)$ of $\hat{E}_{k}$ which contains $\xi$. For each finite sequence of positive integers $b_{1}, b_{2}, \ldots, b_{r}$, let $J^{(m)}\left(b_{1}, b_{2}, \ldots, b_{r}\right)$ be the interval $\left\{\left[0 ; b_{1}, b_{2}, \ldots, b_{r}, x\right] ; x \in\left[\frac{m+2}{m+1}, m+1\right]\right\}$.

Since $\operatorname{dim} C_{m}>1-\frac{1}{m \log 2}$, putting $d_{m}:=1-\frac{1}{m \log 2}$, there is $\tau_{m}>0$ such that, for each $\eta$ with $0<\eta \leq \tau_{m}$, we need at least $\eta^{-d_{m}}$ intervals of length at most $4 \eta$ to cover $C_{m}$. For each $j \geq 0$, we take $t_{0}^{(j)}:=0$, and, while $K\left(a_{1}, \ldots, c_{s_{j}+1}^{(j)}, a_{r_{j}+1}, \ldots, a_{r_{j}+t_{i}^{(j)}}\right)<\frac{\tau_{m} \check{n}_{j}}{(m+1)^{4} K_{0}(\delta)}$, we put

$$
\begin{aligned}
t_{i+1}^{(j)}:=\min \left\{t>t_{i}^{(j)} ;\right. & K\left(a_{1}, \ldots, c_{s_{j}+1}^{(j)}, a_{r_{j}+1}, \ldots, a_{r_{j}+t}\right)> \\
& \left.(m+1) \tau_{m}^{-1 / 2} K\left(a_{1}, \ldots, c_{s_{j}+1}^{(j)}, a_{r_{j}+1}, \ldots, a_{r_{j}+t_{i}^{(j)}}\right)\right\} .
\end{aligned}
$$

We define the positive integer $\ell_{j}$ as the largest integer $i$ for which $t_{i}^{(j)}$ was defined above. We then have

$$
\frac{\tau_{m} \check{n}_{j}}{(m+1)^{4} K_{0}(\delta)} \leq K\left(a_{1}, \ldots, c_{s_{j}+1}^{(j)}, a_{r_{j}+1}, \ldots, a_{r_{j}+t_{i}^{(j)}}\right)<\frac{\tau_{m}^{1 / 2} \check{n}_{j}}{(m+1)^{2} K_{0}(\delta)}
$$


Let $u_{0}=0$ and, for $j \geq 1, u_{j}=\sum_{0 \leq i<j}\left(\ell_{i}+2\right)$. We take, for $j \geq 0$,

$$
\hat{I}_{u_{j}}(\xi)=J^{(m)}\left(a_{1}, a_{2}, \ldots, c_{s_{j}}^{(j)}, c_{s_{j}+1}^{(j)}\right)
$$

for $1 \leq i \leq \ell_{j}$

$$
\hat{I}_{u_{j}+i}(\xi)=J^{(m)}\left(a_{1}, a_{2}, \ldots, c_{s_{j}}^{(j)}, c_{s_{j}+1}^{(j)}, a_{r_{j}+1}, \ldots, a_{r_{j}+t_{i}^{(j)}}\right)
$$

and $\hat{I}_{u_{j}+\ell_{j}+1}(\xi)=J^{(m)}\left(a_{1}, a_{2}, \ldots, a_{r_{j+1}}\right)$.

Since $\hat{n}_{k}=\hat{n}_{k+1}^{o(1)}$, for large $k$, we have, for large $j$,

$$
\hat{m}_{u_{j}}=\left\lceil\left(1+\frac{1}{j}\right) \frac{1}{q_{m_{j}}^{2} \Psi\left(q_{m_{j}}\right)}\right\rceil-\left\lceil\frac{1}{q_{m_{j}}^{2} \Psi\left(q_{m_{j}}\right)}\right\rceil=q_{m_{j}}^{\lambda-2+o(1)}, \quad \varepsilon_{u_{j}}=1 / q_{m_{j}}^{2 \lambda-2+o(1)}
$$

(which follows from the estimates of Lemma 3), and

$$
\hat{m}_{1} \ldots \hat{m}_{u_{j}-1}>q_{m_{j}}^{2 d_{m}}
$$

so

$$
\frac{\log \left(\hat{m}_{1} \ldots \hat{m}_{u_{j}-1}\right)}{-\log \left(\hat{m}_{u_{j}} \varepsilon_{u_{j}}\right)}>\frac{2 d_{m}}{\lambda}-o(1) .
$$

On the other hand, for $1 \leq i \leq \ell_{j}$, we have

$$
\hat{m}_{u_{j}+i}=O(1), \quad \varepsilon_{u_{j}+i}^{-1}=O\left(q_{m_{j}+1+t_{i}^{(j)}}^{-2}\right)
$$

and

$$
\begin{aligned}
\hat{m}_{1} \ldots \hat{m}_{u_{j}+i-1} & >q_{m_{j}}^{\lambda-2+2 d_{m}}\left(q_{m_{j}+1+t_{i}^{(j)}} / q_{m_{j}+1}\right)^{2 d_{m}} \\
& =q_{m_{j}+1+t_{i}^{(j)}}^{2 d_{m}} q_{m_{j}}^{\lambda-2+2 d_{m}-2 d_{m}(\lambda-1)-o(1)} \\
& =q_{m_{j}+1+t_{i}^{(j)}}^{2 d_{m}} q_{m_{j}}^{-\left(2 d_{m}-1\right)(\lambda-2)-o(1)} \\
& >q_{m_{j}+1+t_{i}^{(j)}}^{2 d_{m}-\left(2 d_{m}-1\right)(\lambda-2) /(\lambda-1)-o(1)}=q_{m_{j}+1+t_{i}^{(j)}}^{\left(2 d_{m}+\lambda-2\right) /(\lambda-1)-o(1)},
\end{aligned}
$$

(the estimates of $\hat{m}_{u_{j}+\ell_{j}+1}, \varepsilon_{u_{j}+\ell_{j}+1}$ and $\hat{m}_{1} \ldots \hat{m}_{u_{j}+\ell_{j}}$ are roughly the same as those of $\hat{m}_{u_{j}+\ell_{j}}, \varepsilon_{u_{j}+\ell_{j}}$ and $\left.\hat{m}_{1} \ldots \hat{m}_{u_{j}+\ell_{j}-1}\right)$, which gives

$$
\frac{\log \left(\hat{m}_{1} \ldots \hat{m}_{u_{j}+i-1}\right)}{-\log \left(\hat{m}_{u_{j}+i} \varepsilon_{u_{j}+i}\right)}>\frac{2 d_{m}+\lambda-2}{2(\lambda-1)}-o(1) .
$$

By Proposition 1 of [2], it follows that

$$
\operatorname{dim} \hat{\mathcal{C}} \geq \min \left\{\frac{2 d_{m}}{\lambda}, \frac{2 d_{m}+\lambda-2}{2(\lambda-1)}\right\}=\frac{2 d_{m}}{\lambda}
$$


and, letting $m$ tend to infinity, we conclude that $\operatorname{dim} \operatorname{Exact}(\Psi) \geq 2 / \lambda$, and so $\operatorname{dim} \mathcal{K}(\Psi)=$ $\operatorname{dim} \operatorname{Exact}(\Psi)=2 / \lambda$. This proves Theorem 3 .

Remark. In the proof of Theorem 3, our aim was to construct a Cantor type set, whose Hausdorff dimension could be bounded from below by means of the mass distribution principle, as in [2]. The basic strategy was to construct real numbers $\xi$ whose continued fraction expansion has scattered big partial quotients (which guarantee that $\xi$ is in Exact $(\Psi)$ ) and whose other partial quotients are at most equal to $m$. The method developed in [2] is quite complicated and makes use of the assumption that $x \mapsto x^{2} \Psi(x)$ is non-decreasing, which is much stronger than our assumption $(*)$, to allow the partial quotients to be unbounded, but 'not too big'. The advantage is that it also gives, see Theorem 2 of [2], precise information on the Hausdorff measure of sets related to $\operatorname{Exact}(\Psi)$. In the present paper, our mere goal is simply to compute the Hausdorff dimension of $\operatorname{Exact}(\Psi)$. To do this, it was sufficient to take the 'small' coefficients bounded, say by a large integer $m$. Comparing our result with the construction of [2], what we obtain is analogous to show, with the notation of [2], page 182, that there exists $\varepsilon(m)$ which tends to 0 as $m$ tends to infinity

and is such that, at step $k$, each interval $U_{j}$ gives birth to $Q_{k+1}^{2-\varepsilon(m)} \Psi\left(Q_{k}\right)$ intervals, which are approximately evenly spaced. Letting then $m$ tend to infinity gave us the expected dimension. However, this approach is too crude to give any information on the Hausdorff measure of $\operatorname{Exact}(\Psi)$.

Acknowledgements. This work was started while the first author was visiting the I.M.P.A. He wishes to express his gratitude for the invitation.

\section{References}

[1] V. Beresnevich, H. Dickinson and S. L. Velani, Sets of exact "logarithmic order" in the theory of Diophantine approximation, Math. Ann. 321 (2001), 253-273.

[2] Y. Bugeaud, Sets of exact approximation order by rational numbers, Math. Ann. 327 (2003), 171-190.

[3] Y. Bugeaud, Sets of exact approximation order by rational numbers II, Unif. Distrib. Theory 3 (2008), 9-20.

[4] T. W. Cusick and M. E. Flahive, The Markoff and Lagrange Spectra. Mathematical Surveys and Monographs, vol. 30, American Mathematical Society, Providence, RI, 1989.

[5] H. Dickinson, A note on the theorem of Jarnik-Besicovitch, Glasgow Math. J. 39 (1997), 233-236.

[6] M. M. Dodson, Hausdorff dimension, lower order and Khintchine's theorem in metric Diophantine approximation, J. reine angew. Math. 432 (1992), 69-76.

[7] R. J. Duffin and A. C. Schaeffer, Khintchine's problem in metric Diophantine approximation, Duke J. 8 (1941), 243-255. 
[8] K. Falconer, Fractal Geometry : Mathematical Foundations and Applications. John Wiley \& Sons, 1990.

[9] G. Harman, Metric Number Theory. LMS Monographs New Series, vol. 18, Clarendon Press, 1998.

[10] V. Jarník, Zur metrischen Theorie der diophantischen Approximationen, Práce Mat.Fiz. $36(1928 / 29), 91-106$.

[11] V. Jarník, Über die simultanen Diophantische Approximationen, Math. Z. 33 (1931), 505-543.

[12] A. Ya. Khintchine, Einige Sätze über Kettenbrüche, mit Anwendungen auf die Theorie der diophantischen Approximationen, Math. Ann. 92 (1924), 115-125.

[13] A. Ya. Khintchine, Continued Fractions. University of Chicago Press, 1964.

[14] J. Kurzweil, A contribution to the metric theory of diophantine approximations, Czechoslovak Math. J. 1 (1951), 149-178.

[15] A. V. Malyshev, Markov and Lagrange spectra (survey of the literature), Studies in number theory (LOMI), 4. Zap. Naučn. Sem. Leningrad. Otdel. Mat. Inst. Steklov. (LOMI) 67 (1977), 5-38, 225 (in Russian); English translation in J. Soviet Math. 16 (1981), 767-788.

[16] C. G. Moreira, Geometric properties of the Markov and Lagrange spectra, Preprint.

[17] C. A. Rogers, Hausdorff Measures. Cambridge University Press, Cambridge, 1970.

[18] J. Wu and J. Xu, The distribution of the largest digit in continued fraction expansions, Math. Proc. Cambridge Philos. Soc. 146 (2009), 207-212.

Yann Bugeaud

Université de Strasbourg

U. F. R. de mathématiques

7, rue René Descartes

67084 STRASBOURG (France)

e-mail : bugeaud@math.unistra.fr
Carlos Gustavo Moreira

IMPA

Estrada Dona Castorina 110 RIO DE JANEIRO (Brasil)

gugu@impa.br 